\title{
Root Traits Responses to Irrigation Intervals in Rice (Oryza sativa)
}

\author{
Mahmoud M. Gaballah', Adel M. Ghoneim ${ }^{2}$, Mohamed I. Ghazy ${ }^{1}$, Hassna M. Mohammed ${ }^{3}$, Raghda M. Sakran ${ }^{1}$, \\ Hafeez Ur Rehman ${ }^{4 *}$ and Noraziyah Abd Aziz Shamsudin ${ }^{5}$ \\ ${ }^{1}$ Rice Research and Training Center (RRTC), 33717, Sakha, Kafr El-Sheikh, Egypt \\ ${ }^{2}$ Field Crops Research Institute, Agricultural Research Center (ARC), 12619, Giza, Egypt \\ ${ }^{3}$ Department of Agronomy, Faculty of Agriculture, Kafr El-Sheikh University, Kafr El-Sheikh, Egypt \\ ${ }^{4}$ Department of Agronomy, University of Agriculture, Faisalabad, Pakistan \\ ${ }^{5}$ Department of Biological Sciences and Biotechnology, Faculty of Science and Technology, Faculty of Science and \\ Technology, Universiti Kebangsaan Malaysia, 43600 Bangi, Selangor, Malaysia \\ *For correspondence: hafeezcp@gmail.com; h.rehman@uaf.edu.pk \\ Received 13 February 2021; Accepted 03 April 2021; Published 10 June 2021
}

\begin{abstract}
Drought is one of major abiotic stresses that effect rice production. Roots play vital role in absorption of water and nutrients from soil contributing for drought tolerance. The present study quantified the effects of different irrigation intervals on root development and agronomical traits of three Egyptian rice cultivars, Giza177, Giza178, Sakha107, IET1444 as a popular drought tolerant and Moroberekan as control genotype. Irrigation treatments were imposed 15 days after transplanting and applied for every 4, 8 and 12 days during 2018 and 2019 rice growing seasons. The results showed the reduction in root architecture traits with prolonged irrigation intervals. A significant decrease in plant height, number of panicles plant ${ }^{-1}$, grain yield $\left(\mathrm{t} \mathrm{ha}^{-1}\right)$ and relative water content, while sterility $(\%)$ and water use efficiency significantly increased over irrigation intervals. The highly significant and positive correlation was found among grain yield and root:shoot ratio, relative water content and number of panicles plant ${ }^{-1}$, while the negative correlation was with root xylem vessel number and sterility. It was concluded that, the drought reduced the grain yield and its components due to poor developed root system. Moroberekan and IET1444 genotypes can be used as a donor parent for rice breeding program. Further studies are also required to identify factors that contribute to the high yield potential of both Giza178 and Sakha107 under different water stress condition. (C) 2021 Friends Science Publishers
\end{abstract}

Keywords: Cultivars; Drought; Grain yield; Irrigation regimes; Root traits

\section{Introduction}

Rice (Oryza sativa L.) is staple of more than 3.5 billion people to obtain $20 \%$ of their daily calorie intake. Water is essential for growth and development of rice plants (Yang 2012; Ghoneim 2020). More than $75 \%$ of the world rice is produced under continuous flooding practices (Van et al. 2001). Rice production area in Egypt changes yearly based on the available irrigation water and occupies about $20 \%$ with the total cultivated area of 660 thousand hectares with the total production of 5.5 million tons. About one-third of total cultivated area is exposed to water shortage annually in Egypt (Abdallah et al. 2016). Hence, irrigation water is the most limiting factor for expanding rice cultivation area in Egypt.

Breeding for drought tolerance in rice can be a sustainable approach to reduce the adverse effects of drought stress. Drought tolerance can be assessed through morphological, physiological and agronomical traits
(Farooq et al. 2009; Hussain et al. 2018). Rice roots play a crucial role in the understanding of water stress, its acquisition, water stress adaptation and tolerance (Geng et al. 2018). Considerable variation in root traits is regulated by multiple genes and many studies report that selection for root traits in improving drought tolerance. For instance, roots with increasing penetration rate have an advantage for moisture absorption from deeper soil layers (Hussain et al. 2019). Increased rooting depth, root shoot ratio, root density, root pulling force and penetration ability through hardpans contribute to drought tolerance and have a direct association with the rice root systems (Upriser et al. 2004; Clark et al. 2011; Hazman and Brown 2018).

Likely, xylem vessels play an essential role in plant adaptation to drought stress. Root anatomical features of xylem vessels, including the extraction of nutrients and water from the soil, have a significant impact on plant function and therefore of major importance in understanding plant adaptation to drought stress (Bhugra et al. 2017).

To cite this paper: Gaballah MM, AM Ghoneim, MI Ghazy, HM Mohammed, RM Sakran, HU Rehman and NAA Shamsudin (2021). Root traits responses to irrigation intervals in rice (Oryza sativa). Intl J Agric Biol 26:22-30 
The root length with surface area can determine the uptake of soil resources and young root tips are the key regions of water absorbed. The diameter of xylem vessels affects the hydraulic conductivity of the root and eventually determines the productivity of the plant under drought stress. Breeding strategies to decrease the root xylem diameter can lead to a decrease in hydraulic conductance under adequate accessibility of moisture (Kim et al. 2020). Drying of the soil surface layer could lead the roots to search deep in the soil profile for available moisture.

The water resources in Egypt are limited to the share flow of the Nile River by 55.5 billion $\mathrm{m}^{3}$, the deep groundwater in the deserts and small amounts of rainfall in the northern coastal area. Egypt has pioneered various water-saving irrigation technologies to achieve more waterefficient irrigation for rice and deficit irrigation is most practiced. In this method, soil is dried out to some degree in between irrigation intervals (Ghoneim 2020) and has been effective breeding method for plants with lower root length density in shallow layers of soil and high root length density in medium and deep layers. The hierarchical structure of the root system could promote hydraulic lift, helping water uptake from deep soil profiles. Large-diameter xylem vessels may be useful in raising the axial hydraulic conductivity of roots growing in deep soil layers if deep root systems may increase crop productivity (Kim et al. 2020). Relative water content (RWC), water use efficiency (WUE) and panicle characteristics of rice genotypes are multivariate traits in response to varying degrees of water stress (ChaUm et al. 2010).

Drought stress at the reproductive stage can cause huge impacts on yield and its components. Extreme water stress during grain-filling stage accounts for 48-94\% economic yield losses. If drought stress develops soon after panicle initiation, the number of spikelets developed are declined and this may result in reduction of yield (Sharma et al. 2018; Ikmal et al. 2021). Water scarcity has also been reported to delay or earlier the appearance of panicle and flowering (Shamsudinet al. 2016a, b; Kang and Futakuchi 2019; Ikmal et al. 2019). Panicle length, number of spikelets per panicle and grain yield are significantly reduced by drought (Abdel-Hafez et al. 2017).

Therefore, production of cultivars with both high yield potential and tolerant to drought are key objectives of the rice breeding program. Until today, many drought tolerant rice cultivars have been introduced for increasing productivity per unit area under drought as well as normal conditions, such as BRRI Dhan-56 and -57 (Bangladesh), Hanhui3T (China), Sahbhagi dhan (India), Sukha dhan-1, -2 and -3 (Nepal), Sahod ulan-3 and Katihan-8 (Philippines), and MRIA1, MNR151 and MNR152 (Malaysia) (Ahmed et al. 2016; Li et al. 2018; Sobri et al. 2020). Hence, this study was carried out to understand the changes in root and other morphophysiological traits induced by irrigation interval and to determine the most important criteria for effective selection of drought tolerant rice genotypes.

\section{Materials and Methods}

\section{Plant materials}

Five rice genotypes including three Egyptians cultivars, namely Giza177, Giza178, Sakha107, IET1444 from India and Moroberekan from Republic of Guinean were used in this study. The pedigree information and types of the rice genotypes are presented in (Table 1).

\section{Experimental site and soil properties}

A field experiment was conducted at Rice Research and Training Centre, located at Kafr EL-Sheikh Governorate (31 $09^{\circ} \mathrm{N}$ Latitude and $3068^{\circ}$ longitude) during 2018 and 2019 growing seasons. The air temperature $\left({ }^{\circ} \mathrm{C}\right)$, relative humidity $(\mathrm{RH}, \%)$ and evaporation $\left(\mathrm{mm} \mathrm{day}^{-1}\right)$ during the 2018 and 2019 growing seasons are presented in (Table 2). Representative soil samples were taken in bulk from 0-20 $\mathrm{cm}$ and $20-40 \mathrm{~cm}$ depth before the growing season. The soil samples were air-dried, ground and passed through 2-mm sieve. Composite soil samples were taken and analyzed for physical and chemical characteristics of the soil including electrical conductivity $(\mathrm{EC},) \mathrm{pH}$, organic matter (OM), $\mathrm{CaCO}_{3}$, cations and ions following the standard methods (Page et al. 1982). The physico-chemical characteristics of the soil are given in (Table 3).

\section{Experimental design and treatments}

Field experiment was carried out in a strip-plot design using three replications. The main plots were devoted to the three irrigation intervals, 4, 8, and 12 days with $6 \mathrm{~cm}$ water depth ahead, while five rice genotypes were allocated to subplots. The horizontal plots were surrounded by deep ditches to prevent any lateral movement of water. Pre-germinated seeds were sown on $1^{\text {st }}$ May in both growing seasons. The 28 days old seedlings of all genotypes were transplanted at inter-row distance of $20 \mathrm{~cm}$ with one seedling per hill. Nitrogen fertilizer was applied at $165 \mathrm{~kg} \mathrm{ha}^{-1}$ as urea $(50 \%$ as basal, $30 \%$ at initial tillering and $20 \%$ at panicle initiation). Phosphorus fertilizer was applied at $36 \mathrm{~kg} \mathrm{P}_{2} \mathrm{O}_{5}$ $\mathrm{ha}^{-1}$ using superphosphate $\left(15.5 \% \mathrm{P}_{2} \mathrm{O}_{5}\right)$ as basal during soil preparation. The irrigation intervals were imposed at 15 days after transplanting till harvesting. Water pump was used to irrigate the experiment and amount of water applied throughout the experiment was measured. Water use efficiency (WUE) was calculated as follows:

$$
\text { WUE }=\text { Grain yield }(\mathrm{kg}) / \text { amount of applied water }\left(\mathrm{m}^{3}\right)
$$

Relative water content (RWC) was calculated for flag leaf using following formula:

$$
\text { RWC }(\%)=((F W)-(\text { DW }) /(\text { TW }- \text { DW })) * 100
$$

Where, FW is flag leaf fresh weight, DW is flag leaf dry weight and TW is flag leaf turgid weight. 
Table 1: Pedigree, origin, type and some remarks of the studied genotypes

\begin{tabular}{llll}
\hline Genotypes & Pedigree & Origin & Type \\
\hline Giza177 & Giza 171/Yomji No.1 & Egypt & Japonica \\
& // Pi No. 4 & & \\
Giza178 & Giza 175/Milyang 49 & Egypt & Indica/Japonica \\
Sakha107 & Giza 177 x BL1 & Egypt & Japonica \\
IET1444 & (TN 1/CO 29) & India & Indica \\
Moroberekan & IR 8-24-6-(M307 H5) & Republic of Guinean Japonica \\
\hline
\end{tabular}

Table 2: Monthly relative humidity $(\mathrm{RH}, \%)$, temperature $\left({ }^{\circ} \mathrm{C}\right)$, and Pan evaporation $\left(\mathrm{mm} \mathrm{day}{ }^{-1}\right)$ recorded during the 2018 and 2019 rice growing seasons

\begin{tabular}{|c|c|c|c|c|c|c|}
\hline \multirow[t]{2}{*}{ Months } & \multicolumn{2}{|c|}{ Relative humidity (\%) } & \multicolumn{4}{|c|}{ Temperature $\left({ }^{\circ} \mathrm{C}\right)$ Pan evaporation $\left(\mathrm{mm} \mathrm{d}^{-1}\right)$} \\
\hline & 2018 & 2019 & 2018 & 2019 & 2018 & 2019 \\
\hline May & 44.9 & 44.2 & 26.1 & 26.2 & 6.49 & 6.63 \\
\hline June & 50.1 & 51.9 & 28.3 & 28.1 & 6.78 & 6.89 \\
\hline July & 53.3 & 53.1 & 28.3 & 28.7 & 6.14 & 6.35 \\
\hline Aug. & 59.0 & 58.7 & 30.2 & 30.8 & 5.19 & 5.49 \\
\hline Sep. & 56.4 & 56.4 & 27.5 & 27.2 & 3.17 & 3.18 \\
\hline
\end{tabular}

Table 3: Pre-sowing physico-chemical analysis of experimental soil

\begin{tabular}{|c|c|c|c|c|}
\hline \multirow[t]{2}{*}{ Property } & \multicolumn{2}{|c|}{2018} & \multicolumn{2}{|c|}{2019} \\
\hline & $0-20 \mathrm{~cm}$ & $20-40 \mathrm{~cm}$ & $0-20 \mathrm{~cm}$ & $20-40 \mathrm{~cm}$ \\
\hline $\mathrm{EC}\left(\mathrm{dS} \mathrm{m}^{-1}\right)$ & 2.00 & 2.10 & 2.00 & 2.20 \\
\hline $\mathrm{pH}$ & 8.20 & 8.30 & 8.00 & 8.10 \\
\hline $\mathrm{OM}(\%)$ & 1.30 & 1.30 & 1.20 & 1.20 \\
\hline $\mathrm{CaCO}_{3}(\%)$ & 3.70 & 3.10 & 3.80 & 3.20 \\
\hline Soluble ions $\left(\mathrm{meq} \mathrm{L}^{-1}\right)$ : & & & & \\
\hline $\mathrm{Ca}^{++}$ & 5.10 & 4.80 & 5.40 & 5.20 \\
\hline $\mathrm{Mg}^{++}$ & 2.10 & 2.00 & 2.40 & 2.30 \\
\hline $\mathrm{Na}^{+}$ & 12.00 & 13.10 & 11.8 & 12.30 \\
\hline $\mathrm{K}^{+}$ & 0.40 & 0.50 & 0.60 & 0.50 \\
\hline $\mathrm{HCO}_{3}^{-}$ & 3.50 & 3.80 & 3.70 & 4.20 \\
\hline $\mathrm{Cl}^{-}$ & 14.80 & 14.90 & 15.20 & 15.90 \\
\hline $\mathrm{SO}_{4}^{-}$ & 1.30 & 1.70 & 1.20 & 1.90 \\
\hline Available-P $\left(\mathrm{mg} \mathrm{kg}^{-1}\right)$ & 12.60 & 12.00 & 14.20 & 14.30 \\
\hline Available- $\mathrm{Zn}\left(\mathrm{mg} \mathrm{kg}^{-1}\right)$ & 0.69 & 0.70 & 0.88 & 0.80 \\
\hline Available-Fe $\left(\mathrm{mg} \mathrm{kg}^{-1}\right)$ & 5.20 & 5.10 & 6.10 & 6.00 \\
\hline Available-Mn $\left(\mathrm{mg} \mathrm{kg}^{-1}\right)$ & 2.10 & 2.30 & 2.50 & 2.10 \\
\hline
\end{tabular}

\section{Assessment of root parameters}

Root traits were measured using five plants/genotype at 24 days after stress imposition. A $38 \mathrm{~mm}$ (inner diameter) steel tube was placed next to a hill with less than $1 \mathrm{~cm}$ between the nearby tiller and the tube. The soil column was sampled at $45 \mathrm{~cm}$ deep, collected and cut the soil to a depth of 0-45 $\mathrm{cm}$. Soil samples were placed on $1 \mathrm{~mm}$ mesh screen and roots were washed to take out soil using tap water (Pantuwan et al. 1997). Roots were dried in an oven at $70^{\circ} \mathrm{C}$ for $48 \mathrm{~h}$ and weighed to record dry weight. Root length was measured from the base of the plant to the tip of the main axis of primary root. Root volume $\left(\mathrm{cm}^{3}\right)$ was measured by water displacement technique by placing all the roots in a measuring cylinder and obtaining the displaced water volume. Number of roots plant $^{-1}$ was assessed by the counting roots. Root:shoot ratio, percentage of the root dry weight $(\mathrm{g})$ to the shoot dry weight $(\mathrm{g})$. Root thickness was the average thickness $(\mathrm{mm})$ of the tip portion (about $1 \mathrm{~cm}$ from the tip) of three random secondary roots at the mid position of the root plant ${ }^{-1}$.

\section{Root xylem and its area measurement}

Root sample of two $\mathrm{cm}$ was taken between $1 \mathrm{~cm}$ and $3 \mathrm{~cm}$ from the nodal root tip for each root. The samples were immediately subjected for fixation and storage to FAA (formalin at $10 \%$ volume, acetic acid at $5 \%$ volume, ethyl alcohol at 50\% volume and distilled water at $35 \%$ volume). Root samples were dehydrated with 50, 70 and $95 \%$ ethanol in subsequent steps. The paraffin system was used for penetration and embedding, which was followed by sectioning, removal of xylem and alcohol paraffin. Every embedded root was placed in a microtome which used to cut perpendicular cross sections (10 $\mathrm{mm}$ slice thickness) at a 20 $\mathrm{mm}$ distance from the root tip (Reichert-Jung, Model $1130 /$ Biocut). After staining with safranine and fast green as counter staining (Bhugra et al. 2017), pictures of the root cross sections were taken by a microscope (Olympus BX51) whereby one pixel represented $0.47 \mathrm{~mm}$. The average xylem vessel number of roots was counted under the light microscope. The average xylem vessel diameter was measured under ocular microscope at 10x magnification. The average diameter of all xylem vessels of the three roots/plant were transformed to area by using the formula:

$$
\text { Area }=\pi r^{2}
$$

Where, $\pi=\operatorname{Pi}(3.14), r=$ radius.

\section{Assessment of yield and its components}

At harvesting, rice grain yield was estimated and adjusted to $14 \%$ moisture content. Ten panicles were selected randomly from each plot to measure length of panicle, number of filled and unfilled grains panicle ${ }^{-1}, 100$-grain weight and sterility.

\section{Statistical analysis}

The statistical analysis was done using analysis of variance technique by means of Genes computer software package (Gomez and Gomez 1984). The means of treatment were compared using the Duncan's multiple range test (Duncan 1955). Correlations among these characteristics were also calculated.

\section{Results}

\section{Root traits characteristics}

All root traits were affected significantly by irrigation intervals, rice genotypes and interaction among them (Table 4). The plants irrigated with 4 days interval recorded the highest mean values for all root traits in both rice growing 
Effect of Irrigation Regimes on Rice Roots Traits / Intl J Agric Biol, Vol 26, No 1, 2021

Table 4: Effect of irrigation intervals and rice genotypes on root traits of rice

\begin{tabular}{|c|c|c|c|c|c|c|c|c|c|c|c|c|c|c|}
\hline \multirow[t]{2}{*}{ Treatments } & \multicolumn{2}{|c|}{$\begin{array}{l}\text { Root length } \\
(\mathrm{cm})\end{array}$} & \multicolumn{2}{|c|}{$\begin{array}{c}\text { Root volume } \\
\left(\mathrm{cm}^{3}\right)\end{array}$} & \multicolumn{2}{|c|}{$\begin{array}{c}\text { Number of roots } \\
\text { plant }^{-1}\end{array}$} & \multicolumn{2}{|c|}{ Root: shoot ratio } & \multicolumn{2}{|c|}{$\begin{array}{l}\text { Root thickness } \\
\text { (mm) }\end{array}$} & \multicolumn{2}{|c|}{$\begin{array}{c}\text { Root xylem vessel } \\
\text { number }\end{array}$} & \multicolumn{2}{|c|}{$\begin{array}{c}\text { Root xylem vessel } \\
\text { area }\left(\mathrm{mm}^{2}\right)\end{array}$} \\
\hline & 2018 & 2019 & 2018 & 2019 & 2018 & 2019 & 2018 & 2019 & 2018 & 2019 & 2018 & 2019 & 2018 & 2019 \\
\hline \multicolumn{15}{|c|}{ Irrigation intervals (days) } \\
\hline 4 & $30.64^{\mathrm{a}}$ & $31.01^{\mathrm{a}}$ & $66.68^{\mathrm{a}}$ & $65.77^{\mathrm{a}}$ & $252.00^{\mathrm{a}}$ & $252.53^{\mathrm{a}}$ & $0.77^{\mathrm{a}}$ & $0.76^{\mathrm{a}}$ & $0.86^{\mathrm{a}}$ & $0.87^{\mathrm{a}}$ & $5.35^{\mathrm{a}}$ & $5.36^{\mathrm{a}}$ & $0.25^{\mathrm{a}}$ & $0.26^{\mathrm{a}}$ \\
\hline 8 & $26.60^{\mathrm{b}}$ & $26.32^{\mathrm{b}}$ & $50.04^{b}$ & $50.84^{b}$ & $228.33^{\mathrm{b}}$ & $231.87^{\mathrm{b}}$ & $0.69^{\mathrm{b}}$ & $0.69^{\mathrm{b}}$ & $0.81^{\mathrm{b}}$ & $0.80^{\mathrm{b}}$ & $5.20^{\mathrm{b}}$ & $5.24^{\mathrm{b}}$ & $0.23^{\mathrm{b}}$ & $0.23^{\mathrm{b}}$ \\
\hline 12 & $24.97^{\mathrm{c}}$ & $23.87^{\mathrm{c}}$ & $44.94^{c}$ & $44.57^{\mathrm{c}}$ & $210.67^{\mathrm{c}}$ & $210.80^{c}$ & $0.62^{\mathrm{c}}$ & $0.63^{\mathrm{c}}$ & $0.76^{\mathrm{c}}$ & $0.76^{\mathrm{b}}$ & $5.07^{\mathrm{c}}$ & $5.10^{\mathrm{c}}$ & $0.20^{\mathrm{c}}$ & $0.21^{\mathrm{c}}$ \\
\hline F-Test & & & & & & & & & & & & & & \\
\hline \multicolumn{15}{|l|}{ Rice genotypes } \\
\hline Giza177 & $21.72^{\mathrm{e}}$ & $21.08^{\mathrm{e}}$ & $36.78^{\mathrm{e}}$ & $37.21^{\mathrm{e}}$ & $180.22^{\mathrm{e}}$ & $187.89^{\mathrm{c}}$ & $0.67^{\mathrm{c}}$ & $0.62^{\mathrm{c}}$ & $0.77^{\mathrm{b}}$ & $0.76^{\mathrm{b}}$ & $3.84^{\mathrm{e}}$ & $3.85^{\mathrm{e}}$ & $0.16^{\mathrm{e}}$ & $0.15^{\mathrm{e}}$ \\
\hline Giza178 & $23.70^{\mathrm{d}}$ & $23.20^{\mathrm{d}}$ & $54.79^{\mathrm{c}}$ & $54.19^{\mathrm{c}}$ & $221.00^{\mathrm{c}}$ & $219.89^{\mathrm{b}}$ & $0.71^{\mathrm{ab}}$ & $0.69^{\mathrm{b}}$ & $0.68^{\mathrm{c}}$ & $0.68^{\mathrm{c}}$ & $4.00^{\mathrm{d}}$ & $4.00^{\mathrm{d}}$ & $0.18^{\mathrm{d}}$ & $0.18^{\mathrm{d}}$ \\
\hline Sakha107 & $25.51^{\mathrm{c}}$ & $25.37^{\mathrm{c}}$ & $44.58^{\mathrm{d}}$ & $45.82^{\mathrm{d}}$ & $192.22^{\mathrm{d}}$ & $193.89^{\mathrm{c}}$ & $0.69^{\mathrm{abc}}$ & $0.70^{\mathrm{b}}$ & $0.78^{\mathrm{b}}$ & $0.78^{\mathrm{b}}$ & $4.30^{\mathrm{c}}$ & $4.32^{\mathrm{c}}$ & $0.20^{\mathrm{c}}$ & $0.21^{\mathrm{c}}$ \\
\hline IET1444 & $31.90^{\mathrm{b}}$ & $31.73^{b}$ & $62.07^{\mathrm{b}}$ & $61.53^{\mathrm{b}}$ & $327.44^{\mathrm{a}}$ & $332.33^{\mathrm{a}}$ & $0.72^{\mathrm{a}}$ & $0.77^{\mathrm{a}}$ & $0.68^{\mathrm{c}}$ & $0.69^{c}$ & $5.09^{\mathrm{b}}$ & $5.10^{\mathrm{b}}$ & $0.23^{\mathrm{b}}$ & $0.24^{\mathrm{b}}$ \\
\hline Moroberekan & $34.15^{\mathrm{a}}$ & $33.95^{\mathrm{a}}$ & $71.22^{\mathrm{a}}$ & $69.90^{\mathrm{a}}$ & $230.78^{\mathrm{b}}$ & $224.67^{\mathrm{b}}$ & $0.68^{\mathrm{bc}}$ & $0.69^{\mathrm{b}}$ & $1.14^{\mathrm{a}}$ & $1.15^{\mathrm{a}}$ & $8.83^{\mathrm{a}}$ & $8.85^{\mathrm{a}}$ & $0.37^{\mathrm{a}}$ & $0.38^{\mathrm{a}}$ \\
\hline F-Test & $* *$ & $* *$ & $* *$ & $* *$ & $* *$ & $* *$ & $* *$ & $* *$ & $* *$ & $* *$ & $* *$ & $* *$ & & $* *$ \\
\hline Interaction $(\mathrm{I} \times \mathrm{G})$ & & ns & $* *$ & $* *$ & ** & ** & * & $*$ & $*$ & $*$ & ** & $* *$ & $* *$ & ** \\
\hline
\end{tabular}

Means within a column followed by the same letter do not differ significantly $(P<0.05)$ according to Duncan's Multiple Range Test

$*=$ Significant at $0.05 ; * *=$ Significant at $0.01 ; \mathrm{ns}=$ Non-significant

seasons, while the lowest root traits values were obtained when irrigated at 12 days interval. The highest root traits values including root length, root volume, root thickness, root xylem vessel number and root xylem vessel area were observed for drought tolerant check, Moroberekan while, the IET1444 exhibited superior values for number of roots plant $^{-1}$ and root:shoot ratio in both rice growing seasons. The lowest mean values for all root traits except root thickness were observed for Giza177 while the lowest values were recorded for Giza178 genotype in both rice growing seasons.

Regarding interactive effects, the highest value of root length, root volume, root thickness, xylem vessel number and xylem vessel area were recorded for Moroberekan when irrigated with 4 days interval while the highest number of roots per was observed for IET144 across the two growing seasons. On the other hand, Giza177 under recorded the lowest number of roots plant ${ }^{-1}$ when irrigated at 12 days interval in both growing seasons. The interaction between irrigation intervals and genotypes was also significant for root:shoot ratio. The highest and lowest root:shoot ratio was recorded for IET1444 at 4 days interval and Giza177 at 12 days interval, respectively in both rice growing seasons (Table 5).

\section{Root cross sections}

Root cross sections in indicated the significant differences in root xylem vessel number and area for all genotypes under different irrigation intervals (Fig. 1). All rice genotypes showed xylem vessel number and area decreased by increasing irrigation intervals. The lowest xylem vessel number and area was obtained for Giza177 when irrigated at 12 days interval. In general, least effects of irrigation intervals were observed for Moroberekan thus confirm the high drought tolerant level of this genotype. IET1444 also shown little effects compared to other Egypt rice genotypes under different irrigation intervals. Sakha107 was indicated more tolerance to drought stress compared to Giza178,

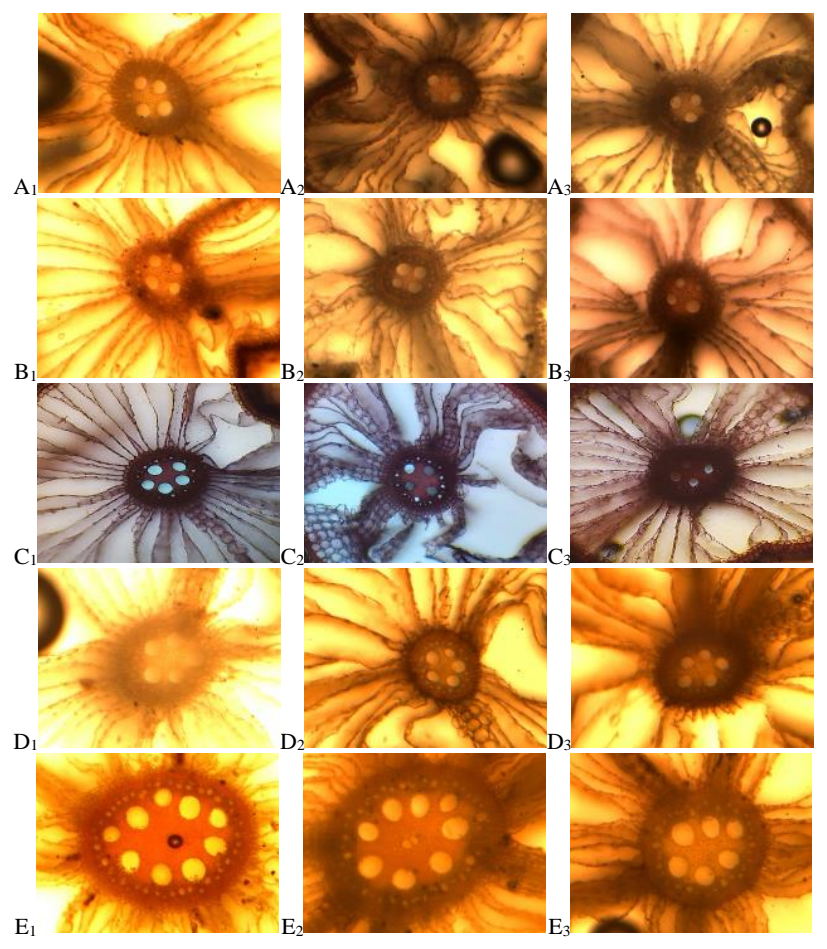

Fig. 1: Rice root cross section illustrated xylem vessel number and area for genotypes under irrigation intervals whereas, $A_{1}, A_{2}$, $A_{3}$ is Giza177; $B_{1}, B_{2}, B_{3}$ is Giza178; $C_{1}, C_{2}, C_{3}$ is Sakha107; $D_{1}$, $\mathrm{D}_{2}, \mathrm{D}_{3}$ is IET1444; $\mathrm{E}_{1}, \mathrm{E}_{2}, \mathrm{E}_{3}$ is Moroberekan at 4,8 and 12 irrigation intervals, respectively

whereas the decrease in root xylem vessel number and area in Sakha107 was lower than Giza178 at 8- and 12days irrigation intervals (Fig. 1).

\section{Relative water content}

The significant effect was found among irrigation intervals on RWC. The results indicated that, RWC decreased with prolonged irrigation intervals in both growing seasons 
Gaballah et al. / Intl J Agric Biol, Vol 26, No 1, 2021

Table 5: Interactive effects of irrigation intervals and rice genotypes on root traits of rice

\begin{tabular}{|c|c|c|c|c|c|c|c|c|}
\hline $\begin{array}{l}\text { Irrigation } \\
\text { (days) }\end{array}$ & intervals Genotypes & $\begin{array}{l}\text { Root length } \\
(\mathrm{cm})\end{array}$ & $\begin{array}{l}\text { Root volume } \\
\left(\mathrm{cm}^{3}\right)\end{array}$ & $\begin{array}{l}\text { Number of roots } \\
\text { plant }^{-1}\end{array}$ & $\begin{array}{l}\text { Root: shoot } \\
\text { ratio }\end{array}$ & $\begin{array}{l}\text { Root thickness } \\
(\mathrm{mm})\end{array}$ & $\begin{array}{l}\text { Root xylem } \\
\text { vessel number }\end{array}$ & $\begin{array}{l}\text { Root xylem vessel } \\
\text { area }\left(\mathrm{mm}^{2}\right)\end{array}$ \\
\hline \multirow[t]{5}{*}{4} & Giza177 & $24.84^{\mathrm{d}}$ & $48.35^{\mathrm{d}}$ & $220.67^{\mathrm{f}}$ & $0.74^{\mathrm{bc}}$ & $0.82^{\mathrm{d}}$ & $4.00^{\mathrm{e}}$ & $0.19^{\mathrm{e}}$ \\
\hline & Giza178 & $27.93^{\mathrm{c}}$ & $63.70^{\mathrm{b}}$ & $257.00^{\mathrm{d}}$ & $0.79^{\mathrm{ab}}$ & $0.7^{\mathrm{e}-\mathrm{h}}$ & $4.00^{\mathrm{e}}$ & $0.19^{\mathrm{e}}$ \\
\hline & Sakha107 & $30.57^{b}$ & $66.78^{b}$ & $208.67^{\mathrm{g}}$ & $0.73^{\mathrm{bc}}$ & $0.83^{\mathrm{d}}$ & $4.60^{\mathrm{d}}$ & $0.22^{\mathrm{d}}$ \\
\hline & IET1444 & $33.19^{\mathrm{b}}$ & $75.38^{\mathrm{a}}$ & $349.67^{\mathrm{a}}$ & $0.82^{\mathrm{a}}$ & $0.71^{\mathrm{e}-\mathrm{h}}$ & $5.17^{\mathrm{c}}$ & $0.25^{\mathrm{d}}$ \\
\hline & Moroberekan & $36.71^{\mathrm{a}}$ & $79.17^{\mathrm{a}}$ & $224.00^{\mathrm{f}}$ & $0.77^{\mathrm{abc}}$ & $1.25^{\mathrm{a}}$ & $9.00^{\mathrm{a}}$ & $0.40^{\mathrm{a}}$ \\
\hline \multirow[t]{5}{*}{8} & Giza177 & $20.96^{\mathrm{fg}}$ & $35.26^{\mathrm{e}}$ & $165.00^{\mathrm{j}}$ & $0.64^{\mathrm{ef}}$ & $0.76^{\text {def }}$ & $3.84^{\mathrm{f}}$ & $0.15^{\mathrm{fg}}$ \\
\hline & Giza178 & $22.8^{\text {def }}$ & $52.06^{\mathrm{d}}$ & $206.00^{\mathrm{g}}$ & $0.70^{\text {cde }}$ & $0.68^{\text {fgh }}$ & $4.00^{\mathrm{e}}$ & $0.17^{\mathrm{f}}$ \\
\hline & Sakha107 & $24.06^{\mathrm{de}}$ & $36.01^{\mathrm{e}}$ & $191.67^{\mathrm{h}}$ & $0.72^{\text {cd }}$ & $0.78^{\mathrm{de}}$ & $4.30^{\mathrm{d}}$ & $0.20^{\mathrm{e}}$ \\
\hline & IET1444 & $31.88^{\mathrm{b}}$ & $58.14^{\mathrm{c}}$ & $332.33^{\mathrm{b}}$ & $0.7^{\text {cde }}$ & $0.67^{\text {fgh }}$ & $5.08^{\mathrm{c}}$ & $0.23^{\mathrm{d}}$ \\
\hline & Moroberekan & $33.3^{\mathrm{b}}$ & $68.75^{\mathrm{b}}$ & $246.67^{\mathrm{e}}$ & $0.66^{\text {def }}$ & $1.14^{\mathrm{b}}$ & $8.84^{\mathrm{ab}}$ & $0.36^{\mathrm{b}}$ \\
\hline \multirow[t]{5}{*}{12} & Giza177 & $19.37^{\mathrm{g}}$ & $26.73^{f}$ & $155.00^{\mathrm{k}}$ & $0.61^{\mathrm{f}}$ & $0.74^{\text {efg }}$ & $3.67^{f}$ & $0.12^{\mathrm{h}}$ \\
\hline & Giza178 & $20.4^{\mathrm{fg}}$ & $48.60^{\mathrm{d}}$ & $200.00 \mathrm{~g}^{\mathrm{h}}$ & $0.63^{\mathrm{f}}$ & $0.66^{\mathrm{gh}}$ & $4.00^{\mathrm{e}}$ & $0.15^{\mathrm{g}}$ \\
\hline & Sakha107 & $21.9^{\text {ef }}$ & $30.94^{\mathrm{ef}}$ & $176.33^{\mathrm{i}}$ & $0.61^{\mathrm{f}}$ & $0.74^{\mathrm{efg}}$ & $4.00^{\mathrm{e}}$ & $0.18^{\mathrm{f}}$ \\
\hline & IET1444 & $30.71^{\mathrm{b}}$ & $52.68^{\mathrm{d}}$ & $300.33^{\mathrm{c}}$ & $0.62^{\mathrm{f}}$ & $0.64^{\mathrm{h}}$ & $5.00^{\mathrm{c}}$ & $0.21^{\mathrm{e}}$ \\
\hline & Moroberekan & $32.45^{\mathrm{b}}$ & $65.75^{\mathrm{b}}$ & $221.67^{\mathrm{f}}$ & $0.61^{\mathrm{f}}$ & $1.04^{\mathrm{c}}$ & $8.67^{\mathrm{b}}$ & $0.33^{\mathrm{c}}$ \\
\hline
\end{tabular}

Means within a column followed by the same letter do not differ significantly $(P<0.05)$ according to Duncan's Multiple Range Test

Table 6: Effect of irrigation intervals and rice genotypes on relative water contents, yield related traits and water use efficiency of rice

\begin{tabular}{|c|c|c|c|c|c|c|c|c|c|c|c|c|}
\hline \multirow[t]{2}{*}{ Treatments } & \multicolumn{2}{|c|}{ RWC (\%) } & \multicolumn{2}{|c|}{ Number of panicles plant $^{-1}$} & \multicolumn{2}{|c|}{ 100-grain weight (g) } & \multicolumn{2}{|c|}{ Sterility $(\%)$} & \multicolumn{2}{|c|}{ Grain yield $\left(\mathrm{t} \mathrm{ha}^{-1}\right)$} & \multicolumn{2}{|c|}{ WUE $\left(\mathrm{kg} \mathrm{m}^{3}\right)$} \\
\hline & 2018 & 2019 & 2018 & 2019 & 2018 & 2019 & 2018 & 2019 & 2018 & 2019 & 2018 & 2019 \\
\hline \multicolumn{13}{|c|}{ Irrigation intervals (days) } \\
\hline 4 & $82.30^{\mathrm{a}}$ & $82.70^{\mathrm{a}}$ & $25.10^{\mathrm{a}}$ & $25.60^{\mathrm{a}}$ & $2.58^{\mathrm{a}}$ & $2.56^{\mathrm{a}}$ & $7.82^{\mathrm{c}}$ & $7.65^{\mathrm{c}}$ & $10.25^{\mathrm{a}}$ & $10.58^{\mathrm{a}}$ & $0.79^{c}$ & $0.77^{\mathrm{c}}$ \\
\hline 8 & $78.40^{\mathrm{b}}$ & $77.80^{\mathrm{b}}$ & $20.10^{\mathrm{b}}$ & $20.30^{\mathrm{b}}$ & $2.42^{\mathrm{b}}$ & $2.45^{\mathrm{b}}$ & $18.10^{\mathrm{b}}$ & $17.65^{\mathrm{b}}$ & $8.57^{\mathrm{b}}$ & $8.64^{\mathrm{b}}$ & $0.84^{\mathrm{b}}$ & $0.83^{\mathrm{b}}$ \\
\hline 12 & $73.60^{\mathrm{c}}$ & $73.60^{\mathrm{c}}$ & $18.30^{\mathrm{c}}$ & $18.60^{\mathrm{b}}$ & $2.31^{\mathrm{c}}$ & $2.32^{\mathrm{c}}$ & $21.60^{\mathrm{a}}$ & $21.02^{\mathrm{a}}$ & $7.77^{\mathrm{c}}$ & $7.72^{\mathrm{c}}$ & $0.91^{\mathrm{a}}$ & $0.91^{\mathrm{a}}$ \\
\hline F-Test & & $* *$ & ** & *** & $* * .01$ & *.* & ** & $* * 1.02$ & $* *$ & $* * 12$ & $* *$ & $* *$ \\
\hline \multicolumn{13}{|l|}{ Rice genotypes } \\
\hline Giza177 & $65.10^{\mathrm{c}}$ & $63.87^{\mathrm{d}}$ & $18.94^{\mathrm{b}}$ & $18.77^{\mathrm{b}}$ & $2.70^{\mathrm{b}}$ & $2.75^{\mathrm{b}}$ & $25.83^{\mathrm{a}}$ & $26.81^{\mathrm{a}}$ & $7.79^{\mathrm{b}}$ & $7.60^{\mathrm{d}}$ & $0.72^{\mathrm{c}}$ & $0.70^{\mathrm{c}}$ \\
\hline Giza178 & $79.23^{\mathrm{b}}$ & $78.13^{\mathrm{c}}$ & $23.13^{\mathrm{a}}$ & $23.13^{\mathrm{a}}$ & $2.22^{\mathrm{c}}$ & $2.21^{\mathrm{c}}$ & $15.09^{\mathrm{b}}$ & $13.99^{\mathrm{b}}$ & $9.38^{\mathrm{a}}$ & $9.67^{\mathrm{a}}$ & $0.91^{\mathrm{a}}$ & $0.87^{\mathrm{b}}$ \\
\hline Sakha107 & $80.80^{\mathrm{b}}$ & $81.36^{\mathrm{b}}$ & $24.00^{\mathrm{a}}$ & $24.86^{\mathrm{a}}$ & $2.60^{\mathrm{b}}$ & $2.66^{\mathrm{b}}$ & $13.40^{\mathrm{c}}$ & $13.55^{\mathrm{b}}$ & $9.32^{\mathrm{a}}$ & $9.43^{\mathrm{b}}$ & $0.91^{\mathrm{a}}$ & $0.95^{\mathrm{a}}$ \\
\hline IET1444 & $81.03^{\mathrm{b}}$ & $81.26^{\mathrm{b}}$ & $22.60^{\mathrm{a}}$ & $22.92^{\mathrm{a}}$ & $2.23^{\mathrm{c}}$ & $2.28^{\mathrm{c}}$ & $13.32^{\mathrm{c}}$ & $12.32^{\mathrm{c}}$ & $7.53^{\mathrm{c}}$ & $7.95^{\mathrm{c}}$ & $0.89^{\mathrm{a}}$ & $0.85^{\mathrm{b}}$ \\
\hline Moroberekan & $84.09^{\mathrm{a}}$ & $85.72^{\mathrm{a}}$ & $17.10^{\mathrm{c}}$ & $18.00^{\mathrm{b}}$ & $3.19^{\mathrm{a}}$ & $3.26^{\mathrm{a}}$ & $11.60^{\mathrm{d}}$ & $10.53^{\mathrm{d}}$ & $7.63^{\mathrm{c}}$ & $7.89^{c}$ & $0.80^{\mathrm{b}}$ & $0.82^{\mathrm{b}}$ \\
\hline F-Test & $* *$ & $* *$ & $* *$ & *** & $* *$ & ** & $* *$ & ** & $* *$ & $* *$ & $* *$ & $* *$ \\
\hline Interaction $\mathrm{I} \times \mathrm{G}$ & $* *$ & $* *$ & $* *$ & * & $* *$ & ** & $* *$ & ** & *** & $* *$ & $* *$ & $* *$ \\
\hline
\end{tabular}

Means within a column followed by the same letter do not differ significantly $(P<0.05)$ according to Duncan's Multiple Range Test

$*=$ Significant at $0.05 ; * *=$ Significant at $0.01 ; \mathrm{ns}=$ Non-significant

(Table 6). Moroberekan and IET1444 genotypes showed the higher RWC while Giza177 showed the lowest RWC in both growing seasons. Highly significant effects of interaction between irrigation intervals and genotypes on RWC were also observed in this study, whereas Moroberekan expressed the highest RWC at 4 days irrigation interval. The lowest RWC were recorded for Giza177 at 12 days irrigation interval in both growing seasons.

\section{Water use efficiency}

Irrigation intervals, rice genotypes and interaction among them has significant effect on WUE of rice in both years of study (Table 6). The highest WUE value was observed at 12 days irrigation interval, while the lowest values were recorded for 4 days irrigation interval in both growing seasons. The higher WUE values were found in Sakha107 and Giza178 indicating that these genotypes had high water productivity under drought stress. Regarding interaction, highest mean value of WUE was recorded for Sakha107 at 12 days irrigation interval. Conversely, the lowest WUE value was observed for Giza177 at 12 days irrigation interval over the two growing seasons (Table 7).

\section{Grain yield and its components}

Irrigation intervals, rice genotypes and interaction among them has significant effect on number of panicles plant ${ }^{-1}$, 100 -grain weight, sterility and grain yield in both years of study (Table 6). Grain yield and its components were significantly decreased under at 12 days irrigation interval in both growing seasons. The highest sterility percent was recorded for at 12 days irrigation interval in both growing seasons, meanwhile the lowest mean values were obtained when irrigated at 4 days interval (Table 6). Giza178 and Sakha107 recorded the highest grain yield and number of panicles plant ${ }^{-1}$ in both growing seasons. Meanwhile, Giza177 produced the highest values of sterility but lowest values of grain yield for both rice growing seasons. Moroberekan recorded lowest values of number of panicle plant $^{-1}$ and sterility, but highest values for 100-grain weight over the two growing seasons. Meanwhile, Giza178 recorded the lowest values of 100-grain weight over the two growing seasons (Table 6).

With respect to interaction among irrigation interval and rice genotypes, the highest number of panicles plant ${ }^{-1}$ was recorded for Giza178 at 4 days irrigation interval, 
Effect of Irrigation Regimes on Rice Roots Traits / Intl J Agric Biol, Vol 26, No 1, 2021

Table 7: Interactive effect of irrigation intervals and rice genotypes on relative water contents, yield related traits and water use efficiency of rice

\begin{tabular}{|c|c|c|c|c|c|c|c|}
\hline $\begin{array}{l}\text { Irrigation } \\
\text { (days) }\end{array}$ & intervals Genotypes & RWC (\%) & $\begin{array}{l}\text { Number of panicles } \\
\text { plant }^{-1}\end{array}$ & $\begin{array}{l}\text { 100-grain weight } \\
(\mathrm{g})\end{array}$ & $\begin{array}{l}\text { Sterility } \\
\text { percentage (\%) }\end{array}$ & Grain yield $\left(\mathrm{t} \mathrm{ha}^{-1}\right)$ & WUE $\left(\mathrm{kg} \mathrm{m}^{3}\right)$ \\
\hline \multirow[t]{5}{*}{4} & Giza177 & $71.63^{f}$ & $24.84^{\mathrm{bc}}$ & $2.39^{\mathrm{d}}$ & $8.56^{\mathrm{g}}$ & $38.63^{\mathrm{cd}}$ & $0.82^{\mathrm{cd}}$ \\
\hline & Giza178 & $82.06^{\mathrm{b}-\mathrm{e}}$ & $26.09^{\mathrm{abc}}$ & $2.25^{\mathrm{hi}}$ & $8.12^{\mathrm{gh}}$ & $46.03^{\mathrm{a}}$ & $0.87^{\mathrm{b}}$ \\
\hline & Sakha107 & $84.53^{\mathrm{abc}}$ & $28.33^{\mathrm{a}}$ & $2.36^{\mathrm{e}}$ & $9.17^{\mathrm{g}}$ & $43.17^{\mathrm{b}}$ & $0.81^{\mathrm{cd}}$ \\
\hline & IET1444 & $84.94^{\mathrm{ab}}$ & $27.44^{\mathrm{ab}}$ & $2.30^{\mathrm{f}}$ & $7.24^{\mathrm{gh}}$ & $40.71^{\mathrm{c}}$ & $0.78^{\mathrm{d}}$ \\
\hline & Moroberekan & $88.06^{\mathrm{a}}$ & $18.68^{\text {fgh }}$ & $3.58^{\mathrm{a}}$ & $6.01^{\mathrm{h}}$ & $36.59^{\mathrm{de}}$ & $0.66^{\mathrm{e}}$ \\
\hline \multirow[t]{5}{*}{8} & Giza177 & $68.67^{f}$ & $17.42^{\mathrm{gh}} \mathrm{i}$ & $2.28^{\mathrm{fg}}$ & $33.09^{\mathrm{b}}$ & $30.50^{\mathrm{h}}$ & $0.68^{\mathrm{e}}$ \\
\hline & Giza178 & $78.88^{\text {de }}$ & $22.04^{\mathrm{de}}$ & $2.23^{\mathrm{ij}}$ & $16.58^{\mathrm{d}}$ & $36.03^{\text {ef }}$ & $0.89^{\mathrm{b}}$ \\
\hline & Sakha107 & $79.67^{\text {cde }}$ & $23.67^{\mathrm{cd}}$ & $2.26^{\mathrm{gh}}$ & $14.29^{\mathrm{e}}$ & $35.45^{\mathrm{efg}}$ & $0.90^{\mathrm{b}}$ \\
\hline & IET1444 & $80.65^{\text {b-e }}$ & $20.66^{\mathrm{ef}}$ & $2.22^{\mathrm{j}}$ & $14.22^{\mathrm{e}}$ & $35.70^{\text {efg }}$ & $0.90^{\mathrm{b}}$ \\
\hline & Moroberekan & $83.87^{\mathrm{a}-\mathrm{d}}$ & $16.63^{\text {hi }}$ & $3.12^{\mathrm{b}}$ & $12.13^{\mathrm{f}}$ & $33.60^{\mathrm{efg}}$ & $0.84^{\text {bc }}$ \\
\hline \multirow[t]{5}{*}{12} & Giza177 & $54.98^{\mathrm{g}}$ & $14.5^{7 \mathrm{i}}$ & $2.10^{1}$ & $35.85^{\mathrm{a}}$ & $24.39^{\mathrm{i}}$ & $0.67^{\mathrm{e}}$ \\
\hline & Giza178 & $76.75^{\mathrm{e}}$ & $21.25^{\text {def }}$ & $2.18^{\mathrm{k}}$ & $20.58^{\mathrm{c}}$ & $30.48^{\mathrm{h}}$ & $0.97^{\mathrm{a}}$ \\
\hline & Sakha107 & $78.19^{\mathrm{e}}$ & $20.00^{\text {efg }}$ & $2.23^{\mathrm{ij}}$ & $16.74^{\mathrm{d}}$ & $33.25^{\text {fgh }}$ & $1.01^{\mathrm{a}}$ \\
\hline & IET1444 & $77.48^{\mathrm{e}}$ & $19.70^{\text {efg }}$ & $2.18^{\mathrm{k}}$ & $18.50^{\mathrm{d}}$ & $34.93^{\mathrm{efg}}$ & $0.99^{\mathrm{a}}$ \\
\hline & Moroberekan & $80.32^{\mathrm{b}-\mathrm{e}}$ & $16.00^{\mathrm{hi}}$ & $2.87^{\mathrm{c}}$ & $16.68^{\mathrm{d}}$ & $32.61^{\mathrm{gh}}$ & $0.91^{\mathrm{b}}$ \\
\hline
\end{tabular}

Means within a column followed by the same letter do not differ significantly $(P<0.05)$ according to Duncan's Multiple Range Test

RWC $=$ Relative water contents; WUE $=$ Water use efficiency

Table 8: Correlation coefficient among yield and root traits

\begin{tabular}{|c|c|c|c|c|c|c|c|c|c|c|c|c|}
\hline Parameters & $\begin{array}{l}\text { Root length } \\
(\mathrm{cm})\end{array}$ & $\begin{array}{l}\text { Root } \\
\text { volume }\left(\mathrm{cm}^{3}\right)\end{array}$ & $\begin{array}{l}\text { No. of roots } \\
\text { plant }^{-1}\end{array}$ & $\begin{array}{l}\text { Root thickness } \\
(\mathrm{mm})\end{array}$ & $\begin{array}{l}\text { Root: } \\
\text { shoot ratio }\end{array}$ & $\begin{array}{l}\text { Root xylem } \\
\text { vessel No. }\end{array}$ & $\begin{array}{l}\text { Root xylem } \\
\text { vessel area }\end{array}$ & $\begin{array}{l}\text { RWC } \\
(\%)\end{array}$ & $\begin{array}{l}\text { No. of panicles } \\
\text { plant }^{-1}\end{array}$ & $\begin{array}{l}100 \text { - grain } \\
\text { weight }(\mathrm{g})\end{array}$ & $\begin{array}{l}\text { Sterility } \\
(\%)\end{array}$ & $\begin{array}{l}\text { Grain yield } \\
\left(\mathrm{t} \mathrm{ha}^{-1}\right)\end{array}$ \\
\hline Root volume $\left(\mathrm{cm}^{3}\right)$ & $0.90^{* *}$ & & & & & & & & & & & \\
\hline Number of roots plant ${ }^{1}$ & $0.66^{* *}$ & $0.61^{* *}$ & & & & & & & & & & \\
\hline Root thickness (mm) & $0.60^{* *}$ & $0.52^{* *}$ & $-0.14^{\mathrm{ns}}$ & & & & & & & & & \\
\hline Root: shoot ratio & $0.63^{* *}$ & $0.66^{* *}$ & $0.64^{* *}$ & $0.08^{\mathrm{ns}}$ & & & & & & & & \\
\hline $\begin{array}{l}\text { Root xylem vessel } \\
\text { number }\end{array}$ & $0.76^{* *}$ & $0.67^{* *}$ & 0.18 & $0.90^{* *}$ & $0.11^{\mathrm{ns}}$ & & & & & & & \\
\hline Root xylem vesselarea & $0.87^{* *}$ & $0.77^{* *}$ & $0.30^{*}$ & $0.88^{* *}$ & $0.33^{*}$ & $0.97^{* *}$ & & & & & & \\
\hline RWC \% & $0.77^{* *}$ & $0.81^{* *}$ & $0.49^{* *}$ & $0.40^{*}$ & $0.63^{* *}$ & $0.54^{* *}$ & $0.69^{* *}$ & & & & & \\
\hline $\begin{array}{l}\text { Number of panicles } \\
\text { plant }^{-1}\end{array}$ & $0.03^{\mathrm{ns}}$ & $0.14^{\mathrm{ns}}$ & $0.39^{*}$ & $-0.49^{* *}$ & $0.70^{* *}$ & $-0.50^{* *}$ & $-0.31^{*}$ & $0.28^{\mathrm{ns}}$ & & & & \\
\hline 100-grain weight (g) & $0.36^{*}$ & $0.23^{\mathrm{ns}}$ & $-0.35^{*}$ & $0.92^{* *}$ & -0.05 & $0.70^{* *}$ & $0.68^{* * *}$ & $0.12^{\mathrm{ns}}$ & $-0.53^{* *}$ & & & \\
\hline Sterility $(\%)$ & $-0.71^{* *}$ & $-0.77^{* *}$ & $-0.53^{\text {** }}$ & $-0.32^{*}$ & $-0.79^{* * *}$ & $-0.38^{*}$ & $-0.57^{* *}$ & $-0.87^{*_{*}}$ & $-0.47^{* *}$ & $-0.11^{\mathrm{ns}}$ & & \\
\hline Grain yield $\left(\mathrm{t} \mathrm{ha}^{-1}\right)$ & $0.02^{\mathrm{ns}}$ & $0.21^{\mathrm{ns}}$ & $0.03^{\mathrm{ns}}$ & $-0.19^{\text {ns }}$ & $0.59^{* *}$ & $-0.37^{*}$ & $-0.18^{\mathrm{ns}}$ & $0.36^{*}$ & $0.81^{* *}$ & -0.20 & $-0.55^{* *}$ & \\
\hline WUE $\left(\mathrm{kg} \mathrm{m}^{3}\right)$ & $-0.11^{\mathrm{ns}}$ & $-0.11^{\mathrm{ns}}$ & $0.10^{\mathrm{ns}}$ & $-0.31^{*}$ & $-0.23^{\text {ns }}$ & $-0.11^{\mathrm{ns}}$ & $-0.12^{\mathrm{ns}}$ & $0.30^{*}$ & $0.08^{\mathrm{ns}}$ & $-0.45^{* *}$ & $-0.21^{\mathrm{ns}}$ & $0.03^{\mathrm{ns}}$ \\
\hline
\end{tabular}

while the lowest number of panicles plant ${ }^{-1}$ was recorded for Moroberekan at 12 days irrigation interval in both growing seasons. Consequently, the heaviest 100-grain weight was observed for Moroberekan at 4 days irrigation interval while the lightest 100-grain weight was recorded for Giza178 at 12 days irrigation interval in both growing seasons. The highest sterility percent was observed for Giza177 at 12 days irrigation interval. On the other hand, the lowest sterility was recorded for Moroberekan at 4 days irrigation interval in both growing seasons (Table 7).

\section{Correlation analysis}

The highly significant positive correlation was found among root length and root volume, number of roots plant ${ }^{-1}$, root:shoot ratio, root thickness, root xylem vessel number, root xylem vessel area, RWC and 100-grain weight (Table 8). The positive and highly significant correlation was shown among root volume and number of roots plant ${ }^{-1}$, root:shoot ratio, root thickness, root xylem vessel number, root xylem vessel area and RWC, whereas the highly negative correlation and significant was found between root volume and sterility. The highly significant positive correlation was found between number of roots plant ${ }^{-1}$ and root:shoot ratio, root xylem vessel area, RWC and number of panicle plant $^{-1}$ while, the negative correlation and significant was found between number of roots plant ${ }^{-1}, 100$ grain weight and sterility. The highly significant positive correlation was illustrated between root thickness and root xylem vessel number, root xylem vessel area, RWC and 100-grain weight, but the highly negative correlation and significant was found between root thickness and number of panicle plant ${ }^{-1}$, sterility and WUE (Table 8).

The highly significant positive correlation was observed among root:shoot ratio and root xylem vessel area, RWC, 100-grain weight and grain yield, therefore, the negative correlation and significant was found with sterility. Root xylem vessel number had highly significant and positive correlation with root xylem vessel area and number of panicles plant $^{-1}$, whereas the negative correlation and significant was shown with number of panicles per plant, sterility and grain yield. Concerning the root xylem vessel area was correlated positive and highly significant with RWC and 100-grain weight, otherwise negative correlated and significant with number of panicles plant ${ }^{-1}$ and sterility. Regarding RWC was correlated positive and significant with 
grain yield and WUE, however, the negative and highly significant correlation was confirmed with sterility (Table 8).

\section{Discussion}

This study focused on root architecture of five rice varieties under different irrigation intervals to understand its relationship with drought tolerance mechanisms. The root traits decreased by increasing irrigation intervals. Rice cultivars irrigated with 12 days interval had the highest negative effect on root length, root volume, number of roots plant $^{-1}$, root:shoot ratio, root thickness, root xylem vessel number and root xylem vessel area. The genotypes performance also varied under water deficit as each genotype had different genetic background. Henry et al. (2012) reported that Aus rice genotype Dular was drought tolerant based on its deep root growth and the highest drought response index.

Most of the root length was extended to 21.08 to 34.15 $\mathrm{cm}$ in top layer of the soil in both Giza177 and Moroberekan, respectively. The restricted root growth in lowland shallow top-soil zones is a result of the hardpan that develops by pudding and maybe due to the limitation of the supply of oxygen in soil depths under anaerobic lowland conditions (Kato et al. 2013). The shallow nature of the root system, genotypic difference in root volume or length is rather limited. Moroberekan and IET1444 had significant higher root length and root volume at $15-30 \mathrm{~cm}$ soil depth and longer than other genotypes. These two genotypes also had desired root traits compared to other three genotypes. Meanwhile, Giza177 with the lowest yield performance also expressed poor root performance of all traits and thus considered as drought susceptible genotype.

It was predicted that high level of drought tolerant can be obtained for rice genotypes with deep root systems than genotypes with shallow roots systems at the $30 \mathrm{~cm}$ deep. According to Ikmal et al. (2019), deep and coarse root is an important avoidance strategy in rice to reduce adverse effect of drought on yield. An enormous root system could be able to extract more water from the soil, but this does not essentially result in higher yield under limited water condition (Sahebi et al. 2018). Larger root system might have resulted in more rapid extraction of available water and therefore, faster development of water shortage could have an adverse effect on grain yield. Moreover, the roots traits like root length, root thickness, root volume, total number of roots, root length density, root dry weight and root:shoot ratio are imperative to induce drought tolerance (Ganapathy et al. 2010). Pushpam et al. (2018) also reported that the drought resistant genotypes had higher root thickness, root volume and deep root system than the sensitive genotypes. Hence, these root characteristics could be utilized for a reliable selection for drought stress.

The results in present study showed the important correlation between RWC, WUE and grain yield and its components performance under different irrigation intervals.
However, the grain yield components decreased gradually by increased water shortage period, also the same trend with RWC while, the WUE increased by increasing irrigation intervals. The RWC reduced under alternative wetting and drying including saturated to one $\mathrm{cm}$ flooding saved about $45 \%$ of fresh water which are similar to alternative wetting and drying over control (Khairi et al. 2015). Under limited water condition, Moroberekan and IET1444 had higher RWC due to well root system that capable to absorb more water from depth soil and maintained water from losses through transpiration.

Interestingly, Giza178 and Sakha107, genotypes without good rooting system compared to Moroberekan and IET1444 showed high yield potential under irrigation treatments. This shows the capability of this genotype to stand well under water limited condition but this capability was not contributed by the root factor. Therefore, further studies should be conducted to identify traits associated to drought resistance mechanism in these genotypes and traits such as stomatal traits, and transpiration and photosynthesis efficiencies should be prioritized. Terra et al. (2010) reported that, the Quebra Cacho cultivar, have the lower drought index and morpho-physiological traits for drought tolerance. The spikelet sterility presented large difference among cultivars, with higher sterility under water stress condition. Remarkably, the 100-grain weight for all genotypes had low response under irrigation intervals. The rice breeders for drought tolerance are concerned to have genotypes with high WUE values companied with high grain yield, this was also observed in this study for Sakha107 and consequently, we recommended using this cultivar for breeding to drought tolerance. Terra et al. (2010) found that lower number of panicles plant ${ }^{-1}$ in some cultivars was noted under water stress conditions. Yield advances under water scarcity condition might occur, even high osmotic adjustment and good root thickness and depth should be combined through breeding.

The correlation coefficient is important factor to identify the relationship among the studied root traits with water status, grain yield and its components under different irrigation treatments. Highly significant and positive correlations between grain yield with root:shoot ratio, RWC and number of panicles plant ${ }^{-1}$ as well as negative correlation of grain yield with sterility indicates that the root system and water relation have direct contribution to drought tolerance and achieved high yield performance under high WUE. Watanabe et al. (2020) illustrated that, significant and positive correlation was found between the root system traits and the surface area but not for the other component roots. Pushpam et al. (2018) and Ikmal et al. (2019) reported that drought resistant genotypes posed higher root volume, root thickness and deep root system than the susceptible genotypes. Furthermore, negative correlation between grain yield and root xylem vessel number and area could indicate that these two root anatomical traits were also associated with drought tolerance in rice. Various studies have also 
reported on the significant effects of root xylem vessel on the water relations and drought resistance in rice (Richards and Passioura 1989; Henry et al. 2012).

\section{Conclusion}

All genotypes responded to drought stress with decreases yield and related traits, and RWC along with increase in sterility and WUE and reduction in root architecture traits when the plants were gradually stressed. Moroberekan and IET1444 have the best root architectural traits and can be manipulated in the development of drought-tolerant rice varieties. In addition, Giza178 and Sakha107 can be classified as drought-tolerant genotypes due to their ability to produce high yield under water stress condition but contributing factors other than root traits should be studied further.

\section{Acknowledgements}

We wish to thank Rice Research and Training Center, Field Crops Research Institute, Agricultural Research Center, Egypt for providing rice genotypes materials and all facilities to complete this work as well as for agronomic management of the experiment.

\section{Author Contributions}

AMG, MIG, HMM and RMS designed and supervised the study, MAG conducted the experiment, collected data and drafted first draft, AMG, HR and NAAS critically reviewed and improved the manuscript.

\section{Conflict of Interest}

The authors declare no competing interests

\section{Data Availability}

The data will be made available on acceptable request to the corresponding author.

\section{Ethics Approval}

Not applicable.

\section{References}

Abdallah AA, SA Badawy, AA Eliba (2016). Response of some root and yield traits to water stress for some rice varieties. J Sustain Agric Sci 42:353-364

Abdel-Hafez AG, AA AbdAllah, MI Ghazy, S El-Degwy (2017). Genetic analysis of water deficit and heat tolerance in rice under Egyptian conditions. Egypt J Plant Breed 21:202-218

Ahmed AU, R Hernandez, F Naher (2016). Adoption of Stress-Tolerant Rice Varieties in Bangladesh. In: Technological and Institutional Innovations for Marginalized Smallholders in Agricultural Development, pp:251-255. Gatzweiler F, JV Braun (Eds.). Springer, Cham, Switzerland
Bhugra SA, S Anupama, B Chaudhury, LA Chugh (2017). Phenotyping of xylem vessels for drought stress analysis in rice. In: 2017 fifteenth IAPR International conference on machine vision applications, pp:428-431. Held on May 8-12, Nagoya University, Nagoya, Japan

Cha-Um S, Y Suravoot, S Kanyaratt (2010). Water deficit stress in the reproductive stage of four indica rice (Oryza sativa L.) genotypes. Pak J Bot 42:3387-3398

Clark RT, RB MacCurdy, JK Jung, JE Shaff, SR McCouch, DJ Aneshansley, LV Kochian (2011). Three-dimensional root phenotyping with a novel imaging and software platform. Plant Physiol 156:455-465

Duncan DB (1955). Multiple range and multiple F tests. Biometrics 11:1-42

Farooq M, A Wahid, O Ito, DJ Lee, KHM Siddique (2009) Advances in drought resistance of rice. Crit Rev Plant Sci 28:199-217

Ganapathy S, SK Ganesh, P Shanmugasundaram, RC Babu (2010). Studies on root traits for drought tolerance in rice (Oryza sativa $\mathrm{L}$.) under controlled (PVC Pipes) condition. Electr J Plant Breed 1:1016-1020

Geng D, P Chen, X Shen, Y Zhang, X Li, L Jiang, Y Xie, C Niu, J Zhang, X Huang, F Ma, Q Guan (2018). Mdmyb88 and Mdmyb124 enhance drought tolerance by modulating root vessels and cell walls in apple. Plant Physiol 178:1296-1309

Ghoneim AM (2020). Soil nutrients availability, rice productivity and water saving under deficit irrigation conditions. J Plant Prod Mans Univ $11: 7-16$

Gomez KA, AA Gomez (1984). Statistical procedures for agricultural research, 2 Edition. John Wiley Sons, New York, USA

Hazman M, KM Brown (2018). Progressive drought alters architectural and anatomical traits of rice roots. Rice 11; Article 62

Henry A, AJ Cal, TC Batoto, RO Torres, R Serraj (2012). Root attributes affecting water uptake of rice (Oryza sativa) under drought. $J$ Exp Bot 63:4751-4763

Hussain M, A Latif, W Hassan, S Farooq, S Hussain, S Ahmad, A Nawaz (2019). Maize hybrids with well-developed root system perform better under deficit supplemental irrigation. Soil Environ 38:203-213

Hussain M, S Farooq, W Hasan, S Ul-Allah, M Tanveer, M Farooq, A Nawaz (2018). Drought stress in sunflower: Physiological effects and its management through breeding and agronomic alternatives. Agric Water Manage 201:152-167

Ikmal AM, AAS Noraziyah, R Wickneswari (2021). Incorporating drought and submergence tolerance QTL in rice (Oryza sativa $\mathrm{L}$.)- the effects under reproductive stage drought and vegetative stage submergence stresses. Plants 10; Article 225

Ikmal AM, Z Nurasyikin, TATNA Riana, ZPD Ellina, R Wickneswari, AAS Noraziyah (2019). Drought yield QTL $(q D T Y)$ with consistent effects on morphological and agronomical traits of two populations of new rice (Oryza sativa L.) lines. Plants 8; Article 186

Kang DJ, K Futakuchi (2019). Effect of moderate drought-stress on flowering time of interspecific hybrid progenies (Oryza sativa $\mathrm{L} . \times$ Oryza Glaberrima Steud.). J Crop Sci Biotechnol 22:75-81

Kato Y, R Tajima, K Homma, A Toriumi, J Yamagishi, T Shiraiwa, P Mekwatanakarn, B Jongdee (2013). Root growth response of rainfed lowland rice to aerobic conditions in northeastern Thailand. Plant Soil 368:557-567

Khairi M, M Nozulaidi, A Afifah, S Jahan (2015). Effect of various water regimes on rice production in lowland irrigation. Aust J Crop Sci 9:153-159

Kim Y, YS Chung, E Lee, P Tripathi, S Heo, KH Kim (2020). Root response to drought stress in rice (Oryza sativa L.). Intl J Mol Sci 21:1-22

Li P, S Ye, H Liu, A Pan, F Ming, X Tang (2018). Cultivation of droughttolerant and insect-resistant rice affects soil bacterial, but not fungal, abundances and community structures. Front Microbiol 9; Article 1390

Page AL, RH Miller, DR Keeney (1982). Methods of Soil Analysis. In: Chemical and Microbiological Properties, pp:539-624. Agron Monograph No. 9

Pantuwan G, S Fukai, M Cooper, JC O'Toole, S Sarkarung (1997). Root traits to increase drought resistance in rainfed lowland rice. In: Breeding Strategies for Rainfed Lowland Rice in Drought-prone Environments, pp:170-179. Fukai, S, M Cooper, J Salisbury (Eds.). Australian Centre for International Agricultural Research, Canberra, Australia 
Gaballah et al. / Intl J Agric Biol, Vol 26, No 1, 2021

Pushpam R, S Manonmani, NV Varthini, S Robin (2018). Studies on yield, root characters related to drought tolerance and their association in upland rice genotypes. Electr J Plant Breed 9:856-862

Richards RA, B Passioura (1989). A breeding program to reduce the diameter of the major xylem vessel in the seminal roots of wheat and its effect on grain yield in rain-fed environments. Aust J Agric Res 40:943-950

Sahebi M, MM Hanafi, MY Rafii, TMM Mahmud, P Azizi, M Osman, R Abiri, S Taheri, N Kalhori, M Shabanimofrad, G Miah, N Atabaki (2018). Improvement of drought tolerance in rice (Oryza sativa L.): Genetics, genomic tools, and the WRKY gene family. BioMed Res Intl 2018; Article 3158474

Shamsudin NAA, BPM Swamy, R Wickneswari, MTS Cruz, R Anitha, A Kumar (2016a). Marker assisted pyramiding of drought yield QTLs into popular Malaysian rice cultivar, MR219. BMC Genet 17; Article 40

Shamsudiin NAA, BPM Swamy, R Wickneswari, MTS Cruz, S Nitika, R Anitha, A Kumar (2016b). Pyramiding of drought yield QTLs into a high-quality Malaysian rice cultivar MRQ74 improves yield under reproductive stage drought. Rice 9; Article 21

Sharma L, M Dalal, RK Verma, SVV Kumar, S Yadav, S Pushkar, SR Kushwaha, A Bhowmik, V Chinnusamy (2018). Auxin protects spikelet fertility and grain yield under drought and heat stresses in rice. Environ Exp Bot 150:9-24
Sobri H, A Rahim, J Harun, AS Mackeste, M Ruzaini, AW Shakinah, S Faiz, A Phua, CKH Shyful, AR Azizi, A Nazrul, AW Latiffah N, AT Anna, LLK Koh, RYM Rafii, I Asma, K Kogeethavani, H Yoshihiro, K Aki, A Noorman, K Rahiniza, H Nor'Aishah, AAS Noraziyah, MH Norainy (2020). Mutation Breeding of Rice for Sustainable Agriculture in Malaysia. Mutation Breeding Project Forum for Nuclear Cooperation in Asia (FNCA) June, 2020, Tokyo, Japan

Terra TGR, TCA Leal, PHN Rangel, HB Barros, AC Santos (2010). Tolerance to drought in rice cultivars in Southern Cerrado area from Tocantins State, Brazil. Acta Sci Agric 32:715-719

Upriser S, T Toojinda, N Udomprasert (2004). Root responses to water deficit under rain-fed lowland rice. Agric Natl Resour 38:448-456

Van DW, R Sakthivadivel, M Renshaw, JB Silver, MH Birley, F Konradsen (2001). Alternate wet/dry irrigation in rice cultivation: A practical way to save water and control malaria and Japanese encephalitis? Research Report 47, 1-30. IWMI, Colombo, Sri Lanka

Watanabe Y, T Kabuki, T Kakehashi, MK Nakata, S Mitsuya, A Yamauchi (2020). Morphological and histological differences among three types of component roots and their differential contribution to water uptake in the rice root system. Plant Prod Sci 23:191-201

Yang CM (2012). Technologies to improve water management for rice cultivation to cope with climate change. Crop Environ Bioinform 8:193-207 